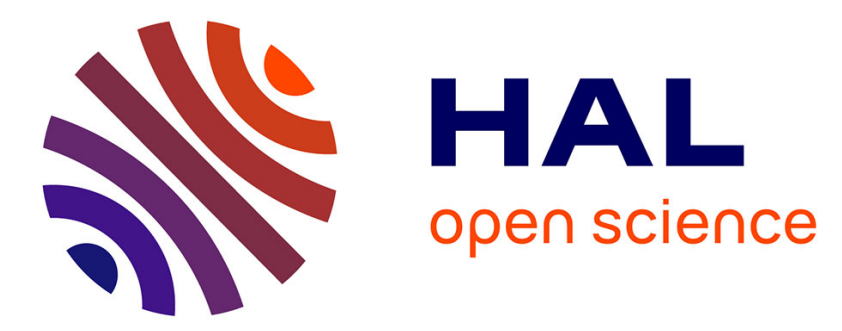

\title{
Strong Enhancement of Terahertz Radiation from Laser Filaments in Air by a Static Electric Field
}

\author{
Aurélien Houard, Yi Liu, Bernard Prade, Vladimir Tikhonchuk, André
}

Mysyrowicz

\section{- To cite this version:}

Aurélien Houard, Yi Liu, Bernard Prade, Vladimir Tikhonchuk, André Mysyrowicz. Strong Enhancement of Terahertz Radiation from Laser Filaments in Air by a Static Electric Field. Physical Review Letters, 2008, 100 (25), pp.255006. 10.1103/PhysRevLett.100.255006 . hal-00455124

HAL Id: hal-00455124

https://hal-polytechnique.archives-ouvertes.fr/hal-00455124

Submitted on 1 Mar 2010

HAL is a multi-disciplinary open access archive for the deposit and dissemination of scientific research documents, whether they are published or not. The documents may come from teaching and research institutions in France or abroad, or from public or private research centers.
L'archive ouverte pluridisciplinaire HAL, est destinée au dépôt et à la diffusion de documents scientifiques de niveau recherche, publiés ou non, émanant des établissements d'enseignement et de recherche français ou étrangers, des laboratoires publics ou privés. 


\title{
Strong Enhancement of Terahertz Radiation from Laser Filaments in Air by a Static Electric Field
}

\author{
Aurélien Houard ${ }^{1}$, Yi Liu ${ }^{1}$, Bernard Prade ${ }^{1}$, Vladimir T. Tikhonchuk ${ }^{2}$, and André Mysyrowicz ${ }^{1 *}$ \\ ${ }^{1}$ Laboratoire d'Optique Appliquée, ENSTA, Ecole Polytechnique, CNRS UMR 7639, Palaiseau, 91761 France \\ ${ }^{2}$ Centre Lasers Intenses et Applications, Université Bordeaux 1, CEA, CNRS UMR 5107, Talence, 33405 France
}

We observe a three order of magnitude enhancement of the terahertz ( $\mathrm{THz}$ ) energy radiated by a femtosecond pulse undergoing filamentation in air in the presence of a static electric field. Measurements of $\mathrm{THz}$ pulse duration, spectrum, polarization and radiation pattern elucidate the physical processes responsible for this radiation. A theoretical model explains the results and predicts another three orders of magnitude enhancement with a TW laser pulse.

The ionization front produced by an intense femtosecond laser pulse propagating in air is the source of a $\mathrm{THz}$ radiation emitted in a narrow cone in the forward direction $[1,2]$. The radiation is produced by the longitudinal oscillations of the plasma left in the wake of the moving ionization front. These plasma oscillations are excited by the ponderomotive force of the laser pulse and are heavily damped by electron collisions on a picosecond timescale. The radiation is emitted by a dipole-like structure moving at the speed of light, it is therefore similar to the Cerenkov emission created by a pair of opposite charges. Because of the simplicity in its implementation, this $\mathrm{THz}$ source is suitable for many applications. It requires no optical element or crystal in the path of the femtosecond laser which would be prone to damage or induce dispersion, and is therefore easily scalable to higher laser intensities. In contrast to other methods such as four wave mixing of femtosecond pulses at frequencies $\omega$ and $2 \omega$, there is no phase sensitive adjustment or precise alignment between pulses. Another attractive feature is the fact that such a source can be placed in the proximity of distant targets, solving thereby the longstanding problem posed by the strong attenuation of $\mathrm{THz}$ radiation in air due to water vapor. Displacing the onset of ionization is easily achieved by either imposing a negative linear chirp to the femtosecond laser pulse or by enlarging the beam diameter $[3,4]$.

In this letter, we show that the THz radiation from the laser filament is highly sensitive to the presence of a transverse electric field. We demonstrate an enhancement of its intensity by three orders of magnitude and show how a further enhancement by at least another three orders of magnitude should be easily obtained. This enhanced source maintains all advantages discussed above since the static field can be also placed in front of the sample. The THz pulse we have obtained in the laboratory with a $2 \mathrm{~mJ}$ femtosecond pulse is already sufficiently intense to allow its complete characterization by nonlinear mixing in air with an infrared source [5]. We present an analytical model explaining all observed features.

A commercial Ti:Sa CPA (chirped pulse amplified) laser chain, delivering 50 fs duration pulses at $800 \mathrm{~nm}$, with a maximum energy of $10 \mathrm{~mJ}$ per pulse operating at a repetition rate of $100 \mathrm{~Hz}$, or a similar system operating at $1 \mathrm{kHz}$ were used. The laser beam was focused in air by a convex lens to form a plasma filament with a typical length of $5 \mathrm{~cm}$. A static electric field (also referred as a DC bias) was applied to the ionized region along direction $X$ (perpendicular to the filament axis $Z$ ) by placing two copper plane electrodes across the filament, see Fig. 1(a). The electrode length was $5 \mathrm{~cm}$ and the maximum applied field was $10 \mathrm{kV} / \mathrm{cm}$. To characterize the $\mathrm{THz}$ radiation emerging from the region between the electrodes, several detectors were used. A bolometric detector cooled to $4 \mathrm{~K}$ in conjunction with a band pass filter centered at $1.3 \mathrm{THz}$ (bandwidth $\sim 1 \mathrm{THz}$ ) measured the $\mathrm{THz}$ pulse energy (see [6] for details). The angular radiation pattern was measured with a heterodyne detector sensitive to the $0.1 \mathrm{THz}$ component of the spectrum [2]. The THz waveform was fully characterized by mixing in ionized air the THz signal with a short laser pulse at $800 \mathrm{~nm}$ to generate an optical signal at $400 \mathrm{~nm}$, using the technique pioneered by Dai et al. [5] (see Fig. 1(b)).

Figure 2 shows the angular patterns of THz radiation measured in the plane $Z$-X, with the detector sensitive to the $X$ component of the polarization. In the absence of static field, we observe the typical conical shape of the $\mathrm{THz}$ emission from the longitudinal current excited by the laser pulse ponderomotive force reported in [2], Fig. 2(a). With increase of the electric field, the energy of THz signal increases, while its peak gradually converges to the filament axis. A change of the DC field polarity reverses the observed pattern with respect to the plane $Z-Y$ (see insets in Fig. 2). We also measured the angular pattern as a function of the filament length, which was varied by changing the focal length of the lens, Fig. 3(a). The influence of the external field on the total energy of $\mathrm{THz}$ signal in the range $0.5-2 \mathrm{THz}$ measured with a bolometer is shown in Fig. 3(b). A quadratic dependence is observed, with no sign of saturation up to the highest applied field of $6 \mathrm{kV} / \mathrm{cm}$. This means that the amplitude of the $\mathrm{THz}$ signal is directly proportional to the static electric field. In another experiment, we obtained a factor $10^{3}$ enhancement with a $2.5 \mathrm{~mJ}$ laser pulse energy and a DC field of $5 \mathrm{kV}$.

The polarization of the $\mathrm{THz}$ signal was measured by rotating a grid polarizer in front of the bolometer around $Z$ axis. The THz emission was found to be linearly polarized in the plane $X-Z$ containing the external electric field, but did not depend on the laser polarization. This excludes a four wave mixing or difference 
frequency generation (DFG) between the laser field and the static field as the origin of the $\mathrm{THz}$ enhancement, as suggested in ref. [7]. Since the THz field generated by the ponderomotive current is radially polarized [1], the polarization and directionality of the signal in the present experiment indicate a direct $\mathrm{THz}$ emission from the transverse current excited in the plasma column by the external electric field.

The THz temporal waveform has been characterized by the technique shown in Fig. 1(b) using a $\mathrm{kHz}$ laser system. Part of the initial pulse at the wavelength of $800 \mathrm{~nm}$ was mixed with the $\mathrm{THz}$ pulse emerging from the filament, yielding a signal at the second harmonic frequency, $400 \mathrm{~nm}$. By measuring the harmonic signal as a function of the delay between the $\mathrm{THz}$ and the ultrashort infrared pulse, one determines the waveform of the $\mathrm{THz}$ field, giving access to the pulse duration and spectrum. This detection method presents the advantage over classical electro-optics sensors to have a better time response since it avoids phonon absorption and dispersion of crystals in the THz range [8]. An example of such a signal is shown in Fig. 4(a). The THz pulse is short, essentially a single cycle. Its spectrum, obtained by a Fourier transform is shown in Fig. 4(b).

We model this laser-created filament as a plasma channel of a cylindrical shape, with a homogeneous electron density $n_{e}$ and a radius $\rho_{c}$. The static external electric field $E_{s}$ is directed along the $X$-axis perpendicularly to the channel axis $Z$. Electrons in the channel feel the electric field $E_{s}$ at the moment they are detached from atoms by the AC electric field of the laser pulse. Therefore the electron motion at different positions $z$ in the channel is similar but occurs with time delay $z / c$, which depends on the distance of a particular cross section from the channel head. That is, the electron motion depends on the coordinates in the channel cross section, $x, y$, and the local time $\tau=t-z / c$.

The plasma in the channel being a good conductor, the electrons start moving under the action of the external electric field, accumulate charge at the channel edge and eventually screen completely the electric field in the plasma. However, there is a transient process of redistribution of charges, and the temporal behaviour of the electric current depends on relation between three parameters: the duration of the ionization process $t_{\text {ion }}$, the electron collision time $t_{\mathrm{col}}$, and the period of plasma oscillations $t_{\mathrm{pl}}$. For the experimental set of parameters of the laser filament in air at normal pressure, the electron density in the channel is $10^{16}-10^{17} \mathrm{~cm}^{-3}$, the channel radius $30-50 \mu \mathrm{m}$ and the laser pulse duration is about $50 \mathrm{fs}$. Then the plasma period is approximately $1-0.5 \mathrm{ps}$, the collision time is of the same order $1-0.3 \mathrm{ps}$; both of them longer than the ionization time. One can therefore consider a plasma response to an instantaneous ionization. The electric current is directed along the direction of the external electric field and it exhibits oscillations at the plasma frequency decaying in a few electron collision times.

The temporal behaviour of the electric current $j_{x}(\tau)$ is qualitatively similar to that in the wake field created by the laser ponderomotive force considered in ref. [2],

$$
j_{x}(\tau)=j_{0} \sin \left(\omega_{p} \tau\right) \mathrm{e}^{-v_{e} \tau / 2}
$$

Here $\omega_{p}=\left(\omega_{p e}^{2}-v_{e}^{2} / 4\right)^{1 / 2}$ is the frequency of the damped plasma oscillation, $\omega_{p e}=\left(e^{2} n_{e} / m_{e} \varepsilon_{0}\right)^{1 / 2}$ is the plasma frequency and $v_{e}$ is the electron collision frequency. There are two major differences from the wake plasma current discussed in ref. [2]: first the current is directed along the external electric field perpendicularly to the channel axis and located inside the circle of the radius $\rho_{0}$; second, its amplitude, $j_{0}=\varepsilon_{0} E_{s} \omega_{p e}^{2} / \omega_{p}$, is proportional to the external field $E_{s}$.

The spectrum of the emitted energy in the solid angle $d \Omega$ is given by:

$$
\frac{d^{2} W}{d \omega d \Omega}=\frac{\left|j_{\omega}\right|^{2}}{4 \pi \varepsilon_{0} c} \frac{\rho_{0}^{4} \cos ^{2} \theta}{(1-\cos \theta)^{2}} \sin ^{2}\left(\frac{L \omega}{2 c}(1-\cos \theta)\right)
$$

The maximum of the emission is directed along the filament axis, $\theta=0$, and the filament length $L$ defines the angular width of the emission cone, $\Delta \theta=4 \pi c / L \omega$. Moreover, in difference from the longitudinal current, the total emitted energy is proportional to the channel length $L$. As shown in Fig. 3(a), the measurements at $0.1 \mathrm{THz}$ are in good agreement with this expression. We observe a linear increase of the radiated energy, and a reduction of the angular width, while increasing the plasma length.

The spectrum of the electromagnetic emission is defined by the current Fourier component:

$$
j_{\omega}=\frac{\varepsilon_{0} E_{s} \omega_{p e}^{2}}{\omega^{2}-\omega_{p e}^{2}+i v_{e} \omega}=\varepsilon_{0} E_{s} g_{\omega}(\omega)
$$

It has a maximum at the plasma frequency and the line width is proportional to the electron collision frequency. The spectrum obtained from the waveform measurement is in a good agreement with this formula (see Fig. 4 (b)). Let us consider also the waveform of the emission at the distance $r>L$ from the source,

$$
E(r, t)=E_{s} \frac{\rho_{0}^{2} \omega_{p}}{r c \theta^{2}} \sin \left(\frac{L \omega_{p}}{4 c} \theta^{2}\right) \cos \omega_{p}\left(t-\frac{r}{c}\right) \mathrm{e}^{-v_{e} t / 2}
$$


This is a typical damped oscillation at the plasma frequency. The on-axis amplitude is proportional to the filament length and the plasma density, and the cone opening is a few degrees for the present parameters.

The analysis of the far field $\mathrm{THz}$ emission provides a method for measuring the ponderomotive potential (or the laser intensity in the filament). For a low DC field, the THz field generated by the ponderomotive force, $E^{l}$ is comparable with the one induced by the static transverse field $E^{2}$. By observing the far field angular pattern resulting from their superposition, one can estimate the relative strength of the two fields, and deduce the ponderomotive potential of the laser pulse responsible for the transition Cerenkov emission. The Fourier components of the two radiation electric fields at the distance $r$ from the filament within the emission cone read:

$$
\left\{\begin{array}{l}
E_{\omega}^{1}(r)=\frac{U_{p} \omega_{p e} g_{\omega} \rho_{0}^{2} \sin (\theta)}{e c^{2} r \theta^{2}} \sin \left(\frac{L \omega}{4 c} \theta^{2}\right) \\
E_{\omega}^{2}(r)=\frac{E_{s} g_{\omega} \rho_{0}^{2}}{r \varepsilon_{0} c \theta^{2}} \sin \left(\frac{L \omega}{4 c} \theta^{2}\right)
\end{array}\right.
$$

where $U_{p}=e^{2} I_{L} /\left(2 m_{e} \varepsilon_{0} c \omega_{0}^{2}\right)$ is the ponderomotive potential proportional to the laser pulse intensity [2]. We compared the calculated pattern at $\omega=0.1 \mathrm{THz}$ with the measurements presented in Fig. 2(b) and (c). The best fit is obtained for $U_{\mathrm{p}}=5.4 \mathrm{eV}$, corresponding to a laser intensity inside the filament $I_{\mathrm{L}}=9 \mathrm{x} 10^{13} \mathrm{~W} \cdot \mathrm{cm}^{-2}$.

The model predicts also a correct dependence of the emitted energy on the DC field. Indeed, the total emitted energy reads:

$$
W \approx \frac{\pi^{2}}{8} \varepsilon_{0} E_{s}^{2} \rho_{0}^{4} L \frac{\omega_{p e}^{3}}{c^{2} v_{e}}
$$

It is proportional to the square of the filament cross section, to the square of the DC electric field strength, to the filament length and to the plasma density in the channel in the power $3 / 2$.

Löffler et al. were the first to report a large enhancement of the THz emitted by ionized air in presence of a static field [9]. Our model of THz emission in a certain sense is similar to the current surge idea they suggested. The emission is produced by an electric current propagating in the wake of the laser pulse. However, the important difference is the predicted coherent and nonlocal nature of the emission, which defines its good directivity and a relatively high radiated energy. There is also very close similarity with the electromagnetic emission from double filament structure [10]. The qualitative features are very similar since the current source has the same direction; the main difference is that the electric field generated by bifilamentation depends on the distance between the filaments, which is not well known. The geometry with external electric field offers better defined conditions and consequently a more unambiguous comparison with a theoretical model.

The model reproduces all observed results. This is true for the radiation pattern for different field strength (as shown by red curves in Fig. 2) and for different plasma column lengths (Fig. 3). The model also predicts the correct dependence of the THz intensity as a function of the DC electric field (see Fig 3(b)). The total emitted energy, measured with the bolometric detector and the band pass filter (see ref. [6] for the calibration method), is estimated to be $0.05 \mathrm{~nJ}$ for $E_{s}=5 \mathrm{kV} / \mathrm{cm}$, laser energy $2.4 \mathrm{~mJ}$ and $L=2 \mathrm{~cm}$, while Eq. (6) for the same parameters gives $0.08 \mathrm{~nJ}$. There are clear possibilities for improvement of the THz emission efficiency. First, one can apply a higher DC electric field. A voltage of $20 \mathrm{kV}$, still well below the threshold for spontaneous sparking discharge, will lead to a factor 16 enhancement. Increasing the distance over which the electric field is applied to $L=20 \mathrm{~cm}$ can bring another factor of 10 . Another improvement by one or two orders of magnitude can be achieved with the use of a more powerful femtosecond laser. Above the threshold of $\sim 10 \mathrm{GW}$, the IR femtosecond pulse generates $N$ filaments, which all act as a source of THz emission (Here, $N \sim P / P_{\mathrm{cr}}$ and $P_{\mathrm{cr}}=5$ $\mathrm{GW}$ is the critical power for filamentation [4]). In the experiment without static field, we observed a factor 40 enhancement of the $\mathrm{THz}$ signal by using a pulse energy of $200 \mathrm{~mJ}$ from the teramobile instead $10 \mathrm{~mJ}$ [2]. Furthermore, since the THz radiation emitted by each individual filament is coherent, by phase sensitive control of the filament pattern one should obtain a THz intensity scaling like $N^{2}$. Altogether, one can expect an increase of the $\mathrm{THz}$ intensity by at least $10^{3}$, bringing the pulse energy content to hundreds of $\mathrm{nJ}$ for a pulse duration of 1 ps.

One can also notice that according to the theoretical model, the same enhancement of the THz generation should be observed if the static field is replaced by a $\mathrm{THz}$ or a microwave pulse of the same electric field (several $\mathrm{kV} / \mathrm{cm}$ ). Indeed, if the period of the applied field is much longer than the time during which ionization occurs $\left(t_{i o n} \approx 50 \mathrm{fs}\right.$ ), this field is seen as static by the ionization front. Thus, by selecting a microwave pulse which is not absorbed by air and focusing it on the filament, one should be able should allow to remotely enhance the $\mathrm{THz}$ emission of the plasma string.

In conclusion, it is demonstrated experimentally that the $\mathrm{THz}$ emission from a femtosecond laser filament in air can be greatly enhanced when an electric field is applied in the ionized air region. A theoretical model has been developed, which explains well all observed features. From far field $\mathrm{THz}$ measurements, most of the 
intrinsic characteristics of the plasma column can be deduced or alternatively the (remote) local electric field can be measured if the plasma properties are known.

The authors thank G. Rey and E. Papalazarou from LOA, G. Gallot from Ecole Polytechnique and B. Leriche from IAS for their experimental support and fruitful discussions. Partial support by a grant from CNRS and DGA is acknowledged.

*Electronic address: andre.mysyrowicz@ensta.fr.

[1] C. D'Amico et al., Phys. Rev. Lett. 98, 235002 (2007).

[2] C. D'Amico et al., New Jour. of Phys. 10, 013015 (2008).

[3] W. Liu, et al., Appl. Phys. B 85, 1, 55 (2006).

[4] A. Couairon and A. Mysyrowicz, Phys. Rep. 441, 47 (2007).

[5] J. Dai, X. Xie, and X. -C. Zhang, Phys. Rev. Lett. 97, 103903 (2006).

[6] A. Houard, Y. Liu, A. Mysyrowicz and B. Leriche, Appl. Phys. Lett. 91, 241105 (2007).

[7] K. Reimann, Rep. Prog. Phys. 70, 1597 (2007).

[8] N. Karpowicz et al., Appl. Phys. Lett. 92, 011131 (2008)

[9] T. Löffler, F. Jacob, and H. G. Roskos, Appl. Phys. Lett. 77, 453 (2000).

[10] Y. Liu et al., Phys. Rev. Lett. 99, 135002 (2007).

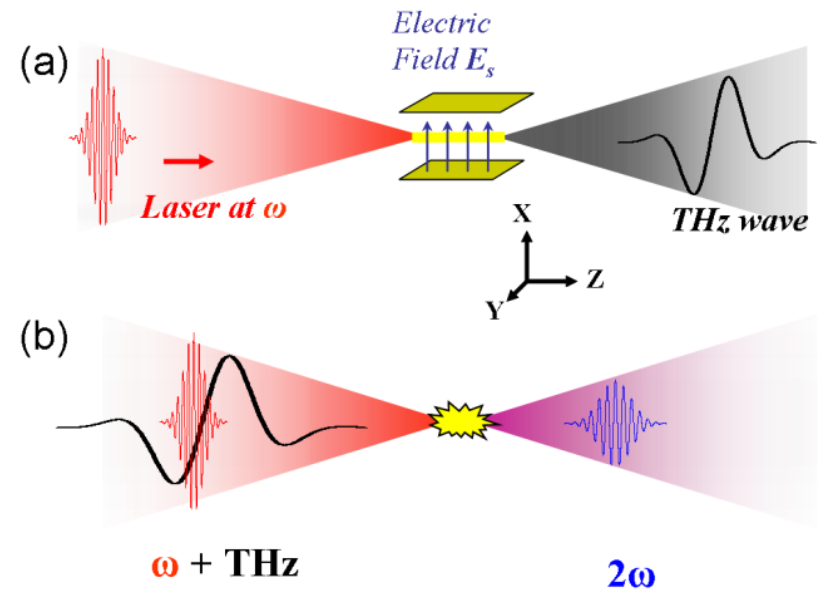

FIG 1. Experimental method for $\mathrm{THz}$ generation (a) and for $\mathrm{THz}$ detection (b).

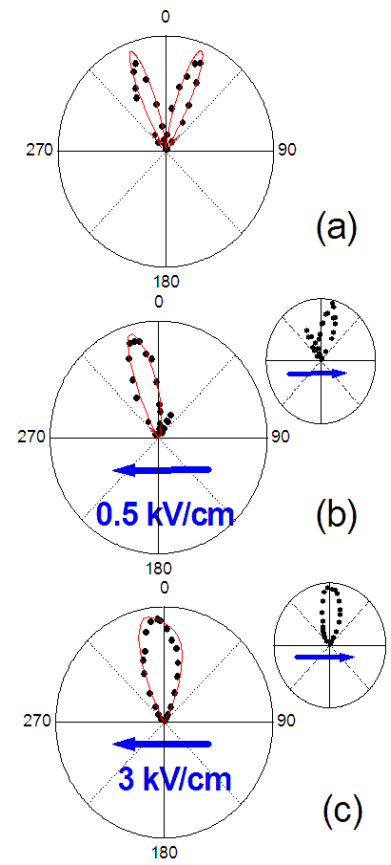


FIG 2. Angular dependence of the THz emission generated in a filament in presence of a DC field $\left|\mathrm{E}_{\mathrm{s}}\right|=0$ (a), 0.5 (b) and 3 $\mathrm{kV} / \mathrm{cm}$. Measurements were made at $0.1 \mathrm{THz}$ with a focusing lens $F=1000 \mathrm{~mm}$ generating a $2 \mathrm{~cm}$ plasma filament. Red continuous curves: fit using Eq. (5) with a laser intensity $I_{\mathrm{L}} \approx 9 \times 10^{13} \mathrm{~W} / \mathrm{cm}^{2}$.
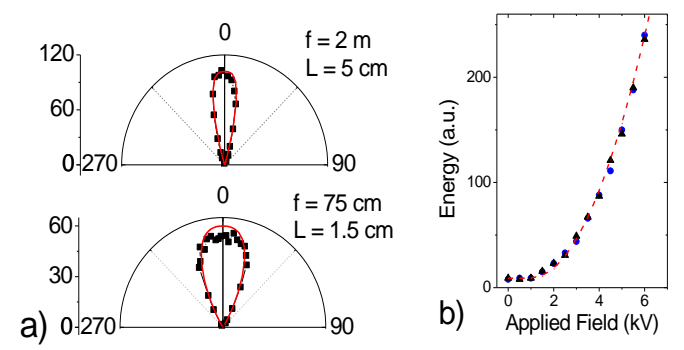

FIG 3. (a) Angular dependence of $\mathrm{THz}$ emission in presence of a static field $\mathrm{E}_{\mathrm{s}}=5 \mathrm{kV} / \mathrm{cm}$ for the filament lengths 1.5 and 5 $\mathrm{cm}$. Red curves are fits using Eq. (2) with the corresponding plasma lengths. (b) Energy of the THz radiation in the range $1-$ $2.5 \mathrm{THz}$ measured as a function of the DC electric field for a $2 \mathrm{~cm}$ plasma. The input IR pulse has energy of $1.4 \mathrm{~mJ}$ and a pulse duration of $50 \mathrm{fs}$.
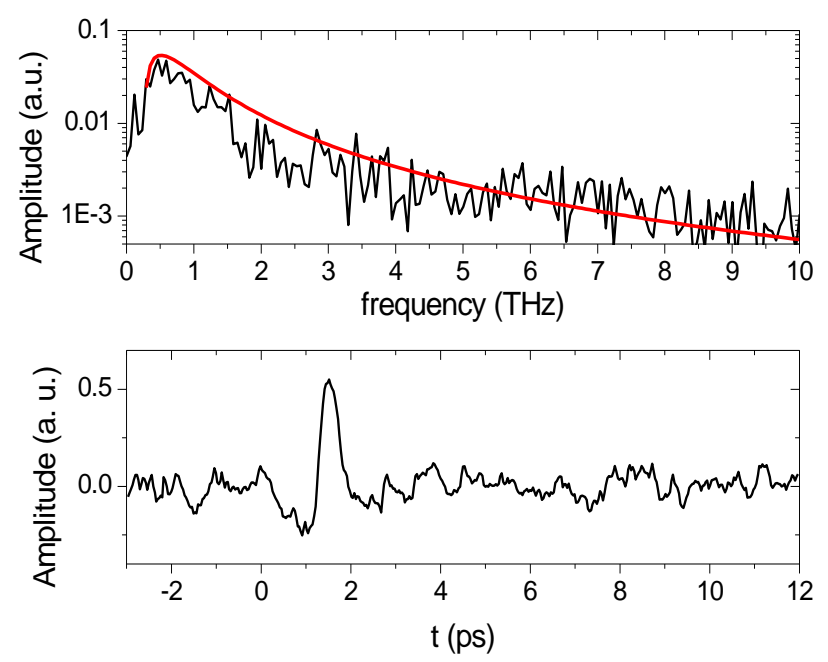

FIG 4. (a) THz field measured by induced second harmonic generation. (b) Corresponding spectrum in the range $0.1-10$ THz obtained by a Fourier transform. The red dotted curve is calculated using Eq. (3) with $\omega_{\mathrm{pe}}=1.9 \times 10^{12} \mathrm{rad} \cdot \mathrm{s}^{-1}$ and $v_{\mathrm{e}}=$ $\left.5 \times 10^{12} \mathrm{rad} . \mathrm{s}^{-1}\right)$. 